\title{
Predictive factors associated with mortality and discharge in intensive care units: a retrospective cohort study
}

Mohammad Ghorbani ${ }^{1}$, Haleh Ghaem ${ }^{2}$, Abbas Rezaianzadeh ${ }^{3}$, Zahra Shayan ${ }^{4}$, Farid Zand ${ }^{5}$, Reza Nikandish ${ }^{6}$

${ }^{1}$ Ph.D. of Epidemiology, Assistant Professor, Department of Public Health, Torbat Heydariyeh University of Medical Sciences, Torbat Heydariyeh, Iran

${ }^{2} \mathrm{Ph}$.D. of Epidemiology, Assistant Professor, Research Center for Health Sciences, Institute of Health, Department of Epidemiology, School of Health, Shiraz University of Medical Sciences, Shiraz, Iran

${ }^{3}$ MD, MPH, Ph.D. of Epidemiology, Professor, Colorectal Research Center, Shiraz University of Medical Sciences, Shiraz, Iran

${ }^{4}$ Ph.D. of Biostatistics, Assistant Professor, Trauma Research Center, Department of Community Medicine, School of Medicine, Shiraz University of Medical Sciences, Shiraz, Iran

${ }^{5}$ M.D., Professor of Anesthesia and Critical Care Medicine, Anesthesiology and Critical Care Research Center, Shiraz University of Medical Sciences, Shiraz, Iran

${ }^{6}$ M.D., Associate Professor of Anesthesia and Critical Care, Department of Emergency Medicine, School of Medicine, Shiraz University of Medical Sciences, Shiraz, Iran

\section{Type of article: Original}

\begin{abstract}
Background and aim: Accurate prediction of prognosis of patients admitted to intensive care units (ICUs) is very important for the clinical management of the patients. The present study aims to identify independent factors affecting death and discharge in ICUs using competing risk modeling.

Methods: This retrospective cohort study was conducted on enrolling 880 patients admitted to emergency ICU in Namazi hospital, Shiraz University of Medical Sciences, Shiraz, Iran during 2013-2015. The data was collected from patients' medical records using a researcher-made checklist by a trained nurse. Competing risk regression models were fitted for the factors affecting the occurrence of death and discharge in ICU. Data analysis was conducted using STATA 13 and R 3.3.3 software.

Results: Among these patients, $682(77.5 \%)$ were discharged and $157(17.8 \%)$ died in the ICU. The patients' mean \pm SD age was $48.90 \pm 19.52$ yr. Among the study patients, $45.57 \%$ were female and $54.43 \%$ were male. In the competing risk model, age (Sub-distribution Hazard Ratio (SHR)) $=1.02,95 \%$ CI: 1.007-1.032), maximum heart rate (SHR=1.009, 95\% CI: 1.001-1.019), minimum sodium level (SHR=1.035, 95\% CI: 1.007-1.064), PH $(\mathrm{SHR}=7.982,95 \% \mathrm{CI}: 1.259-50.61)$, and bilirubin $(\mathrm{SHR}=1.046,95 \% \mathrm{CI}: 1.015-1.078)$ increased the risk of death, while maximum sodium level ( $\mathrm{SHR}=0.946,95 \% \mathrm{CI}$ : $0.908-0.986)$ and maximum $\mathrm{HCT}(\mathrm{SHR}=0.938,95 \% \mathrm{CI}$ : 0.882-0.998) reduced the risk of death.

Conclusion: In conclusion, the results of this study revealed several variables that were effective in ICU length of stay (LOS). The variables that independently influenced time-to-discharge were age, maximum systolic blood pressure, minimum HCT, maximum WBC, and urine output, maximum HCT and Glasgow coma score. The results also showed that age, maximum heart rate, maximum sodium level, $\mathrm{PH}$, urine output, and bilirubin, minimum sodium level and maximum HCT were the predictors of death. Furthermore, our findings indicated that the competing risk model was more appropriate than the Cox model in evaluating the predictive factors associated with the occurrence of death and discharge in patients hospitalized in ICUs. Hence, this model could play an important role in managers' and clinicians' decision-making and improvement of the standard of care in ICUs.

Keywords: Mortality; Discharge; Intensive care units; Competing risk
\end{abstract}

\section{Corresponding author:}

Assistant Professor Dr. Haleh Ghaem, Department of Epidemiology, School of Health, Shiraz University of Medical Sciences, Shiraz, Iran. Tel: +98.7137256007, Fax: +98.7137251006, Email: ghaemh@sums.ac.ir

Received: May 31, 2017, Accepted: September 16, 2017, Published: March 2018

iThenticate screening: September 09, 2017, English editing: March 14, 2018, Quality control: March 15, 2018

This article has been reviewed / commented by three experts

(C) 2018 The Authors. This is an open access article under the terms of the Creative Commons Attribution-NonCommercialNoDerivs License, which permits use and distribution in any medium, provided the original work is properly cited, the use is non-commercial and no modifications or adaptations are made. 


\section{Introduction}

Accurate prediction of prognosis of patients admitted to Intensive Care Units (ICUs) is very important for the patients and their family, assessing the quality of care, clinical management of patients, and predicting the likelihood of readmission (1). Early identification of high-risk patients, permit resources to be used more suitably and prevents avoidable morbidities and deaths $(2,3)$. Over the past few decades, statistical techniques have been increasingly used for modeling risks in health research. In this context, many studies have been carried out on calculating the risk of death before surgery, which has led to the development of a large number of risk scoring systems $(4,5)$. Although advances in surgical techniques and postoperative care have resulted in a gradual decrease in mortality rates (6), no decrease has been observed in morbidity rate after surgery and ICU services (7). Thus, the tendency of researchers has shifted towards evaluation of the factors associated with death and ICU discharge. In recent years, several risk scoring systems have been developed to predict the factors associated with mortality in and discharge from ICUs (810). Unfortunately, most of these system do not have sufficient power and do not present a proper definition of outcomes (7). Therefore, they cannot accurately predict the risk factors associated with mortality in and discharge from ICUs (7). With increase in the tendency towards models of predicting mortality after surgery, many authors have made attempts to make similar methods to assess the factors affecting mortality in and discharge from ICUs. These studies have achieved varying levels of success, but their results were contradictory in most cases (9, 11-18). In these studies, several factors were identified that had an impact on ICU length of stay (LOS). However, they have presented different definitions of both influencing variables and ICU LOS. In addition to different definitions among studies, ICU LOS was considered as a categorical variable, which destroyed the coherence of these studies (19). In case inherently continuous variables are converted to binary variables, detailed prediction may be lost that ultimately reduces the potential practical application (14). On the other hand, some studies considered ICU LOS as a time to event variable, but took death as censored $(7,13)$. Similar to other time-to-event data, ICU LOS requires competing risk analysis framework and specific statistical models $(19,20)$. Competing risk situations occur when a person can experience more than one type of event, and occurrence of an event (death in ICU) prevents the occurrence of another event (discharge from ICU) $(20,21)$. When there are competing risks, Kaplan-Meier estimates cannot be interpreted as probability and, consequently, a specific approach is needed based on cumulative incidence function (22). Competing risk regression models allow us to identify independent risk factors for the two outcomes (death and discharge) and create two different algorithms. The Namazi Center is a tertiary referral hospital that is located in Shiraz (the capital of Fars province) and provides medical services to patients from southern parts of Iran. Clinicians working in ICUs should predict patient outcomes for efficient use of ICU beds rather than treatment and monitoring of critically ill patients. The present study aims to identify independent factors affecting the incidence of death and discharge in ICU using competing risk modeling. The results would be useful for managers' and clinicians' decision-making and improvement of the standard of care in ICUs.

\section{Material and Methods}

This retrospective cohort study was conducted in 2016 to evaluate the factors affecting the occurrence of death and discharge in ICU. According to estimates made by the authorities of ICU, all 880 patients admitted to emergency ICU in Namazi Hospital, Shiraz University of Medical Sciences, Shiraz, Iran from January 2013 to December 2015 were recruited. Inclusion criteria were all patients who were admitted to emergency ICU in Namazi Hospital from January 2013 to December 2015, and were enrolled into the research. No exclusion criteria were considered. The data were collected from the patients' medical records using a researcher-made checklist. The variables included age, sex, dates of admission, discharge, or death, body temperature, systolic and diastolic blood pressure, heart rate, respiratory rate, serum concentrations of sodium, glucose, blood urea nitrogen (BUN), creatinine, and blood hematocrit (HCT) and white blood cells (WBC), urine output during the first 24 hour of ICU stay, serum albumin and bilirubin, fraction of inspired oxygen $\left(\mathrm{FiO}_{2}\right), \mathrm{PH}$, partial pressure of oxygen $\left(\mathrm{PO}_{2}\right)$, partial pressure of carbon dioxide $\left(\mathrm{PCO}_{2}\right)$, bicarbonate $\left(\mathrm{HCO}_{3}\right)$, Glasgow Coma Score, and Acute Physiology, Age, and Chronic Health Evaluation IV (APACHE IV) score. The patients' follow-up of discharge or death until 2015, as failure time, was done by referring to their medical records. Additionally, ICU LOS was determined by subtracting the admission date from discharge, death, or censored date. It should be noted that lost to follow-up patients were considered to be right censored. This study was approved by Shiraz University of Medical Sciences ethics committee (94-7636). Competing risk regression models were fitted for the factors affecting the occurrence of death and discharge in ICU. In this regard, admission to ICU, discharge from ICU, and death were considered to be the starting point of followup, primary outcome, and the only competing risk, respectively. Cumulative incidence function is an important quantity that most physicians are interested in the condition of competing risks. This quantity calculates the probability of occurrence of a specific cause, while specific-cause hazard function calculates the instantaneous rate of an event. The data was gathered from studying the medical records of patients who were admitted to emergency 
ICU by a trained nurse. Patient's medical records were used to obtain the outcome and required information of each patient. At first, the relationship between ICU LOS and the predictors was assessed using log rank test in bivariate analysis. Then, all variables with $\mathrm{p}<0.2$ were entered into the multivariate model. It should be noted that the possible correlations among the predictors were evaluated prior to the multivariate analysis. Cox regression analysis was conducted using STATA 13 and competing risks regression analysis was done under the Fine and Gray model, function crr in the CMPRSK package installed on R software (23). The significance level was set at 0.05 .

\section{Results}

This study was performed on 880 patients. Among these patients, 41 (4.7\%) were right-censored because they had been transferred to another ICU. Indeed, 682 patients $(77.5 \%)$ were discharged and $157(17.8 \%)$ died in the ICU. The patients were followed up for 9,212 days and the median (inter quartile range) of ICU LOS was 6 (11) days. Basic characteristics of the patients have been described in Tables 1 and 2. These variables were tested as possible predictors of ICU LOS. The cumulative incidence of the two outcomes has been illustrated in Figure 1. Accordingly, the function of ICU duration immediately increased over the first days but then tailed off slowly. This rapid increase is due to the high rate of discharge during the first days of ICU stay. In addition, the long right tail reflects the low number of patients with long ICU stay. The median of ICU LOS was 6 days and the $25^{\text {th }}$ and $75^{\text {th }}$ quartiles were 2 and 13 days, respectively. As Figure 1 depicts, time-to-discharge followed different patterns from time-to-death. The results indicated no significant correlations among the predictors. Then, univariate analysis was performed using log-rank and Gray's tests. Based on the results, many variables were significantly associated with discharge and death, which have been presented in Table 3 and Figure 2. A positive Hazard Ratio (HR) or Sub-distribution Hazard Ratio (SHR) means that a variable increases the occurrence of the event of interest. The variables with $p<0.2$ in the univariate analysis were entered into the multivariate model. Based on Table 3 , the results of the Cox model were quite different from those of the competing risk model. In the Cox model, maximum diastolic blood pressure and APS score reduced the risk of death, while minimum respiratory rate and Glasgow coma score increased the risk of death. On the other hand, in the competing risk model where death was considered as the event of interest, age, maximum heart rate, minimum sodium level, $\mathrm{PH}$, and bilirubin increased the risk of death, while maximum sodium level and maximum HCT reduced the risk of death. When discharge was considered as the event of interest, age, maximum systolic blood pressure, minimum HCT, and maximum WBC reduced the probability of discharge, while maximum HCT and Glasgow coma score increased the probability of discharge (Table 4).

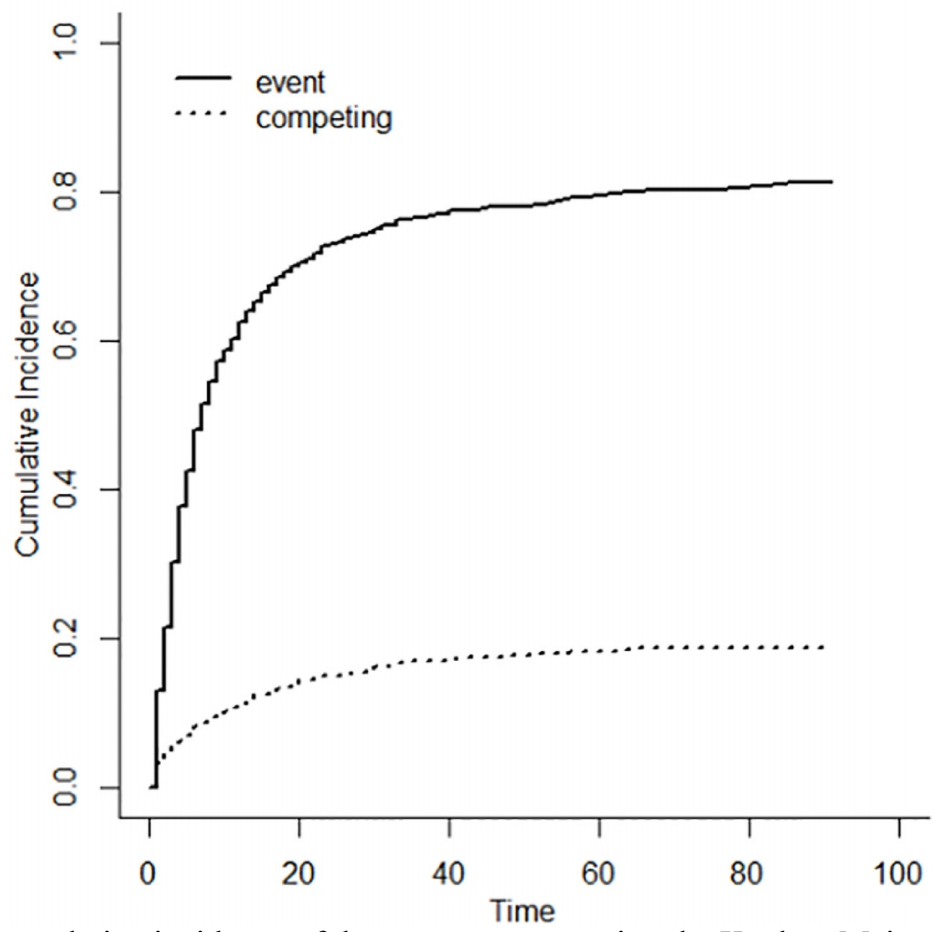

Figure 1. The cumulative incidence of the two outcomes using the Kaplan-Meier estimate (1-KM). 
Table 1. Baseline characteristics of the patients

\begin{tabular}{|c|c|c|}
\hline \multicolumn{2}{|l|}{ Variables } & Mean \pm SD / n (\%) \\
\hline \multicolumn{2}{|l|}{ Age (year) } & $48.90 \pm 19.52$ \\
\hline \multirow[t]{2}{*}{ Sex, n (\%) } & Male & $479(54.43)$ \\
\hline & Female & $401(45.57)$ \\
\hline \multicolumn{2}{|c|}{ Fraction of inspired oxygen $\left(\mathrm{FiO}_{2}\right) \%$} & $52.80 \pm 24.22$ \\
\hline \multicolumn{2}{|c|}{ PH } & $7.38 \pm 0.12$ \\
\hline \multicolumn{2}{|c|}{ Partial pressure of oxygen $\left(\mathrm{PO}_{2}\right)(\mathrm{mmhg})$} & $99.67 \pm 58.63$ \\
\hline \multicolumn{2}{|c|}{ Partial pressure of carbon dioxide $\left(\mathrm{PCO}_{2}\right)(\mathrm{mmhg})$} & $40.02 \pm 15.47$ \\
\hline \multicolumn{2}{|c|}{ Bicarbonate $\left(\mathrm{HCO}_{3}\right)(\mathrm{mEq} / \mathrm{L})$} & $23.80 \pm 7.04$ \\
\hline \multicolumn{2}{|c|}{ Urine output during the first 24 hour of ICU stay $(\mathrm{ml} / 24 \mathrm{hrs})$} & $2996.67 \pm 2292.58$ \\
\hline \multicolumn{2}{|c|}{ Serum Albumin $(g / d L)$} & $3.51 \pm 0.66$ \\
\hline \multicolumn{2}{|c|}{ Bilirubin $(\mathrm{mg} / \mathrm{dL})$} & $1.68 \pm 2.85$ \\
\hline \multicolumn{2}{|c|}{ Potassium $(\mathrm{K})(\mathrm{mEq} / \mathrm{L})$} & $3.94 \pm 0.70$ \\
\hline \multicolumn{2}{|c|}{ Glasgow Coma Score } & $10.36 \pm 4.14$ \\
\hline \multicolumn{2}{|c|}{ APACHE IV score } & $53.15 \pm 29.47$ \\
\hline \multicolumn{2}{|c|}{ APS score } & $51.91 \pm 29.33$ \\
\hline
\end{tabular}

Table 2. Baseline characteristics of the patients (minimum and maximum in the first $24 \mathrm{~h}$ of ICU stay)

\begin{tabular}{|l|l|l|}
\hline Variables & Minimum $($ Mean \pm SD) & Maximum $($ Mean \pm SD) \\
\hline Temperature $\left({ }^{\circ} \mathrm{C}\right)$ & $36.53 \pm 0.92$ & $38.12 \pm 0.88$ \\
\hline Systolic blood pressure $(\mathrm{mm} / \mathrm{Hg})$ & $102.52 \pm 19.11$ & $149.76 \pm 25.47$ \\
\hline Diastolic blood pressure $(\mathrm{mm} / \mathrm{Hg})$ & $60.33 \pm 13.95$ & $93.35 \pm 16.21$ \\
\hline Heart rate $(/ \mathrm{min})$ & $71.21 \pm 18.20$ & $108.09 \pm 22.95$ \\
\hline Respiratory rate $(/ \mathrm{min})$ & $12.63 \pm 5.92$ & $27.39 \pm 10.12$ \\
\hline Sodium $(\mathrm{mEq} / \mathrm{L})$ & $139.08 \pm 4.73$ & $142.30 \pm 6.06$ \\
\hline Glucose $(\mathrm{mg} / \mathrm{dL})$ & $116.88 \pm 51.95$ & $158.94 \pm 89.54$ \\
\hline Creatinine $(\mathrm{mg} / \mathrm{dL})$ & $1.48 \pm 1.87$ & $1.80 \pm 2.43$ \\
\hline Blood urea nitrogen & $23.05 \pm 22.03$ & $27.47 \pm 25.42$ \\
\hline Hematocrit $(\%)$ & $35.91 \pm 8.07$ & $38.66 \pm 8.14$ \\
\hline White blood cells $\left(\times 10^{3} / \mathrm{mm}^{3}\right)$ & $10.72 \pm 5.77$ & $13.16 \pm 8.03$ \\
\hline
\end{tabular}

Table 3. Univariate analysis on the relationship between the study factors and death and discharge

\begin{tabular}{|l|l|l|l|}
\hline \multirow{2}{*}{ Variables } & Cox regression & Competing risk \\
\cline { 2 - 4 } & Discharge HR & Discharge SHR & Death SHR \\
\hline Age & $0.99^{* *}$ & $0.987^{* *}$ & $1.026^{* *}$ \\
\hline Sex (male to female) & 0.952 & 0.964 & 1.191 \\
\hline $\mathrm{FiO} 2(\%)$ & $0.991^{* *}$ & $0.987^{* *}$ & $1.02^{* *}$ \\
\hline $\mathrm{PH}$ & 1.831 & $3.318^{* *}$ & $0.057^{* *}$ \\
\hline $\mathrm{PO}_{2}$ & 1 & 1 & 1 \\
\hline $\mathrm{PCO}_{2}$ & 0.999 & 0.997 & 1.009 \\
\hline $\mathrm{HCO}_{3}$ & 1.01 & $1.02^{* *}$ & $0.955^{* *}$ \\
\hline Urine output & $1.00005^{* *}$ & $1.00005^{*}$ & 0.9998 \\
\hline Albumin & $1.401^{* *}$ & $1.465^{* *}$ & $0.515^{* *}$ \\
\hline Bilirubin & 0.963 & $0.907^{* *}$ & $1.101^{* *}$ \\
\hline $\mathrm{K}$ & 0.952 & 0.957 & 1.133 \\
\hline Glasgow coma score & $1.09^{* *}$ & $1.111^{* *}$ & $0.832^{* *}$ \\
\hline APACHE IV score & $0.984^{* *}$ & $0.978^{* *}$ & $1.027^{* *}$ \\
\hline APS score & $0.984^{* *}$ & $0.979^{* *}$ & $1.029^{* *}$ \\
\hline
\end{tabular}

${ }^{* *} \mathrm{p}<0.01, * \mathrm{p}<0.05$. HR, hazard ratio; SHR, sub-distribution hazard ratio. 

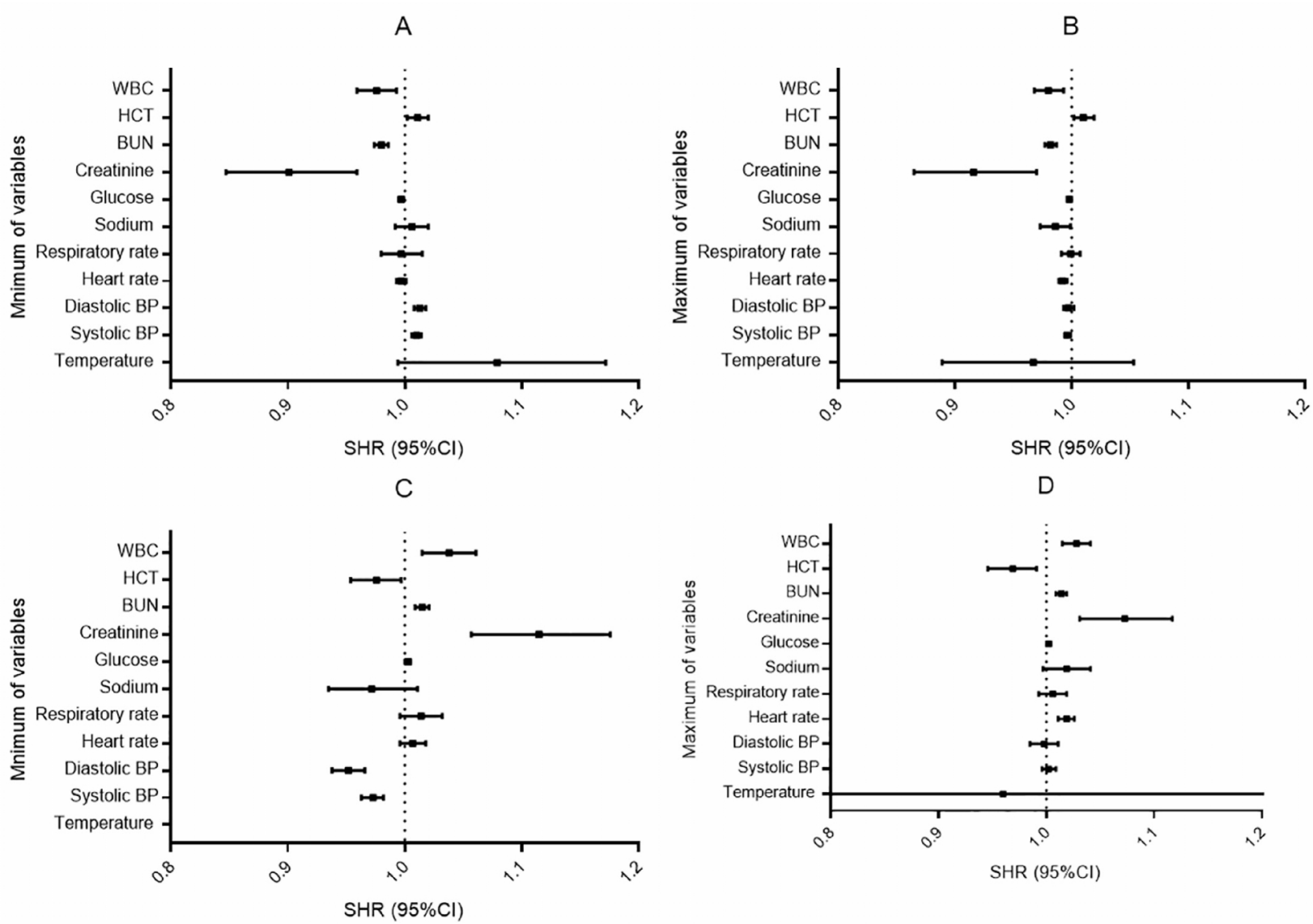

Figure 2. A and B: Sub-distribution hazard ratio $(95 \% \mathrm{CI})$; discharge is the event of interest. C and D: Subdistribution hazard ratio $(95 \% \mathrm{CI})$; death is the event of interest. WBC, white blood cells; HCT, hematocrit blood test; BUN, blood urea nitrogen; BP, blood pressure; HR, hazard ratio; SHR, sub-distributed hazard ratio.

Table 4. Multivariate analysis on the relationship between the study factors and death and discharge

\begin{tabular}{|l|l|l|l|}
\hline \multirow{2}{*}{ Variables } & Cox regression & \multicolumn{1}{l|}{ Competing risk } \\
\cline { 2 - 4 } & Discharge HR & Discharge SHR & Death SHR \\
\hline Age & & $0.994^{*}$ & $1.02^{* *}$ \\
\hline Maximum diastolic blood pressure & $0.988^{* *}$ & & \\
\hline Maximum heart rate & & & $1.009^{*}$ \\
\hline Minimum respiratory rate & $1.017^{*}$ & & \\
\hline Maximum systolic blood pressure & & $0.995^{*}$ & \\
\hline Maximum sodium & & & $0.946^{* *}$ \\
\hline Minimum sodium & & & $1.035^{*}$ \\
\hline Minimum hematocrit & & $0.969^{* *}$ & \\
\hline Maximum hematocrit & & $1.033^{* *}$ & $0.938^{*}$ \\
\hline Maximum white blood cells & & $0.983^{*}$ & \\
\hline PH & & & $7.982^{*}$ \\
\hline Urine output & $1.00006^{*}$ & $1.00007^{* *}$ & $0.9998^{*}$ \\
\hline Bilirubin & & & $1.046^{* *}$ \\
\hline Glasgow coma score & $1.046^{* *}$ & $1.056^{*}$ & \\
\hline APS score & $0.99^{*}$ & & \\
\hline
\end{tabular}

\section{Discussion}

This study aimed to identify the predictive factors associated with the occurrence of death and discharge in patients hospitalized in ICUs using specific statistical analyses (20). In this retrospective cohort study, the following results 
were obtained. Firstly, the results showed that when death was considered censored in the Cox model, maximum diastolic blood pressure, minimum respiratory rate, urine output, Glasgow coma score, and APS score were significant. Accordingly, maximum diastolic blood pressure decreased the probability of discharge, while minimum respiratory rate, urine output, Glasgow coma score, and APS score increased the probability of discharge. In the study conducted by Alison et al., the results of logistic regression analysis revealed that increasing age, APACHE II score, duration of hospital stay before intensive care admission, and discharge Therapeutic Intervention Scoring System (TISS) score were the independent risk factors for death (2). In that study, logistic regression was used for analysis and thus, the results cannot be compared to those of our model. Secondly, when death was not considered censored in the competing risk model, different results were obtained. Accordingly, age, maximum HCT, maximum WBC, urine output, and Glasgow coma score were statistically significant. In other words, maximum HCT and Glasgow coma score increased the probability of discharge, while age, maximum WBC, and urine output decreased the probability of discharge. Thirdly, our results indicated that age, maximum heart rate, maximum sodium level, maximum HCT, and bilirubin had significant effects on death. In the final model in the study conducted by Barili et al., the factors that mostly affected time-to-discharge from ICU were critical preoperative state, emergency status, poor left ventricular dysfunction, and serum creatinine $>200 \mu \mathrm{mol} / \mathrm{L}$. Conversely, the time-to-ICU death model included only two variables, namely critical perioperative state and serum creatinine $>200 \mu \mathrm{mol} / \mathrm{L}$. Up to now, many studies have investigated the risk factors associated with ICU LOS. These studies have achieved varying levels of success, but their results were contradictory in most cases $(9,11-18)$. The results of these studies revealed several effective factors in ICU LOS. Nevertheless, they have presented various definitions of both effective factors and ICU LOS. In addition, ICU LOS was considered as a categorical variable, which destroyed the coherence of these studies (19). In fact, conversion of inherently continuous variables to binary ones can lead to loss of detailed prediction, eventually reducing the potential practical application (14). In the current study, competing risk model was applied. Competing risk regression models allow us to identify independent risk factors for the two outcomes (death and discharge) and create two different algorithms. The findings of the previous studies and clinical practices have demonstrated a relationship between ICU LOS and some variables. Hence, identification of these variables can result in better quality control and pre-operative resources planning, reduce costs, and improve the standard of care in ICUs $(9,11-18)$. Recently, time-to-event data in ICUs were suggested to be analyzed as a competing risk situation. Therefore, if due attention is not paid to the competing risk situation, the effective variables in discharge and death cannot be determined accurately.

\section{Strengths and Limitations of the Study}

One of the strengths of our study was that the large sample size was pursued during a long time period. Additionally, utilization of novel statistical methods resulted in better identification of the factors affecting the occurrence of death and discharge. On the other hand, this study had some limitations. The first study limitation was related to its retrospective design, including the inability to confirm causation and dependence on preciseness of medical records. The second study limitation was enrolment of the patients who were admitted to the emergency ICU, which might restrict the generalizability of the results. Another study limitation was that although all of the HRs are significant, they are small. Therefore, further studies based on greater sample sizes are required to improve the accuracy of models. Finally, the study variables were not repeatedly measured over time to examine their time dependency.

\section{Conclusions}

In conclusion, the results of this study revealed several variables that were effective in ICU LOS. The variables that independently influenced time-to-discharge were age, maximum systolic blood pressure, minimum HCT, maximum WBC, and urine output, which decreased the probability of discharge, and maximum HCT and Glasgow coma score that decreased the probability of discharge. The results also showed that age, maximum heart rate, maximum sodium level, $\mathrm{PH}$, urine output, and bilirubin increased the probability of death, while minimum sodium level and maximum HCT decreased the probability of death. Furthermore, this is the first study in Iran to evaluate the relationship between clinical and socio-demographic factors associated with death and discharge among patients admitted to ICU through applying advanced statistical models. The present study reveals that different clinical and demographic factors were the predictors of death and discharge.

\section{Acknowledgments:}

The present article was extracted from the thesis written by Mohammad Ghorbani and was financially supported by Shiraz University of Medical Sciences, grants number (94-7636). Hereby, the authors would like to thank Ms. A. Keivanshekouh at the Research Improvement Center of Shiraz University of Medical Sciences for improving the use of English in the manuscript. 


\section{Conflict of Interest:}

There is no conflict of interest to be declared.

\section{Authors' contributions:}

All authors contributed to this project and article equally. All authors read and approved the final manuscript.

\section{References:}

1) Min H, Avramovic S, Wojtusiak J, Khosla R, Fletcher RD, Alemi F, et al. A Comprehensive Multimorbidity Index for Predicting Mortality in Intensive Care Unit Patients. J Palliat Med. 2017; 20(1): 35-41. doi: 10.1089/jpm.2015.0392. PMID: 27925837.

2) Campbell AJ, Cook JA, Adey G, Cuthbertson BH. Predicting death and readmission after intensive care discharge. Br J Anaesth. 2008; 100(5): 656-62. doi: 10.1093/bja/aen069. PMID: 18385264.

3) Power GS, Harrison DA. Why try to predict ICU outcomes? Curr Opin Crit Care. 2014; 20(5): 544-9. doi: 10.1097/MCC.0000000000000136. PMID: 25159474.

4) Nashef SA, Roques F, Michel P, Gauducheau E, Lemeshow S, Salamon R. European system for cardiac operative risk evaluation (EuroSCORE). Eur J Cardiothorac Surg. 1999; 16(1): 9-13. doi: 10.1016/S10107940(99)00134-7. PMID: 10456395.

5) Roques F, Nashef SA, Michel P, Gauducheau E, De Vincentiis C, Baudet E, et al. Risk factors and outcome in European cardiac surgery: analysis of the EuroSCORE multinational database of 19030 patients. Eur J Cardiothorac Surg. 1999; 15(6): 816-23. doi: 10.1016/S1010-7940(99)00106-2. PMID: 10431864.

6) Song HK, Diggs BS, Slater MS, Guyton SW, Ungerleider RM, Welke KF. Improved quality and costeffectiveness of coronary artery bypass grafting in the United States from 1988 to 2005 . J Thorac Cardiovasc Surg. 2009; 137(1): 65-9. doi: 10.1016/j.jtcvs.2008.09.053. PMID: 19154905.

7) De Cocker J, Messaoudi N, Stockman BA, Bossaert LL, Rodrigus IE. Preoperative prediction of intensive care unit stay following cardiac surgery. Eur J Cardiothorac Surg. 2011; 39(1): 60-7. doi: 10.1016/j.ejcts.2010.04.015. PMID: 20627608.

8) Herman C, Karolak W, Yip AM, Buth KJ, Hassan A, Legare JF. Predicting prolonged intensive care unit length of stay in patients undergoing coronary artery bypass surgery-development of an entirely preoperative scorecard. Interact Cardiovasc Thorac Surg. 2009; 9(4): 654-8. doi: 10.1510/icvts.2008.199521. PMID: 19640868.

9) Janssen DP, Noyez L, Wouters C, Brouwer RM. Preoperative prediction of prolonged stay in the intensive care unit for coronary bypass surgery. Eur J Cardiothorac Surg. 2004; 25(2): 203-7. doi: 10.1016/j.ejcts.2003.11.005. PMID: 14747113.

10) Tu JV, Jaglal SB, Naylor CD. Multicenter validation of a risk index for mortality, intensive care unit stay, and overall hospital length of stay after cardiac surgery. Steering Committee of the Provincial Adult Cardiac Care Network of Ontario. Circulation. 1995; 91(3): 677-84. PMID: 7828293.

11) Bucerius J, Gummert JF, Walther T, Doll N, Falk V, Schmitt DV, et al. Predictors of prolonged ICU stay after on-pump versus off-pump coronary artery bypass grafting. Intensive Care Med. 2004; 30(1): 88-95. doi: 10.1007/s00134-003-1950-5. PMID: 14504725.

12) Ghotkar SV, Grayson AD, Fabri BM, Dihmis WC, Pullan DM. Preoperative calculation of risk for prolonged intensive care unit stay following coronary artery bypass grafting. J Cardiothorac Surg. 2006; 1(1): 14. doi: 10.1186/1749-8090-1-14. PMID: 16737548, PMCID: 1526720.

13) Hein OV, Birnbaum J, Wernecke K, England M, Konertz W, Spies C. Prolonged intensive care unit stay in cardiac surgery: risk factors and long-term-survival. Ann Thorac Surg. 2006; 81(3): 880-5. doi: 10.1016/j.athoracsur.2005.09.077. PMID: 16488688.

14) Lawrence D, Valencia O, Smith E, Murday A, Treasure T. Parsonnet score is a good predictor of the duration of intensive care unit stay following cardiac surgery. Heart. 2000; 83(4): 429-32.

15) Rosenfeld R, Smith JM, Woods SE, Engel AM. Predictors and outcomes of extended intensive care unit length of stay in patients undergoing coronary artery bypass graft surgery. J Card Surg. 2006; 21(2): 14650. doi: 10.1111/j.1540-8191.2006.00196.x. PMID: 16492273.

16) Tu JV, Mazer CD, Levinton C, Armstrong PW, Naylor CD. A predictive index for length of stay in the intensive care unit following cardiac surgery. CMAJ. 1994; 151(2): 177-85. PMID: 8039063, PMCID: 1336878.

17) Wong DT, Cheng DC, Kustra R, Tibshirani R, Karski J, Carroll-Munro J, et al. Risk factors of delayed extubation, prolonged length of stay in the intensive care unit, and mortality in patients undergoing 
coronary artery bypass graft with fast-track cardiac anesthesia: a new cardiac risk score. Anesthesiology. 1999; 91(4): 936-44. doi: 10.1097/00000542-199910000-00012. PMID: 10519495.

18) Xu J, Ge Y, Hu S, Song Y, Sun H, Liu P. A simple predictive model of prolonged intensive care unit stay after surgery for acquired heart valve disease. J Heart Valve Dis. 2007; 16(2): 109-15. PMID: 17484455.

19) Messaoudi N, De Cocker J, Stockman B, Bossaert LL, Rodrigus IE. Prediction of Prolonged Length of Stay in the Intensive Care Unit After Cardiac Surgery: The Need for a Multi - institutional Risk Scoring System. J Card Surg. 2009; 24(2): 127-33. doi: 10.1111/j.1540-8191.2008.00716.x. PMID: 18793238.

20) Barili F, Cheema FH, Barzaghi N, Grossi C. The analysis of intensive care unit length of stay in a competing risk setting. Eur J Cardiothorac Surg. 2012; 41(1): 232. PMID: 21600782, PMCID: 3241085.

21) Deslandes E, Chevret S. Joint modeling of multivariate longitudinal data and the dropout process in a competing risk setting: application to ICU data. BMC Med Res Methodol. 2010; 10: 69. doi: 10.1186/14712288-10-69. PMID: 20670425, PMCID: 2923158.

22) Klein JP, Andersen PK. Regression modeling of competing risks data based on pseudovalues of the cumulative incidence function. Biometrics. 2005; 61(1): 223-9. doi: 10.1111/j.0006-341X.2005.031209.x. PMID: 15737097.

23) de Wreede LC, Fiocco M, Putter H. mstate: an R package for the analysis of competing risks and multistate models. J Stat Softw. 2011; 38(7): 1-30. doi: 10.18637/jss.v038.i07. 